Research Article

\title{
Determination of Sinomenine in Cubosome Nanoparticles by HPLC Technique
}

\author{
Yanfang Zhou, ${ }^{1}$ Chunlian Guo, ${ }^{1}$ Hongying Chen, ${ }^{1}$ Yudai Zhang, \\ Xinsheng Peng, ${ }^{1}$ and Ping $\mathrm{Zhu}^{2}$ \\ ${ }^{1}$ Guangdong Medical College, Xincheng Avenue, Guangdong 523808, China \\ ${ }^{2}$ Department of Cardiovascular Surgery, Guangdong Cardiovascular Institute, Guangdong General Hospital, \\ Guangdong Academy of Medical Sciences, Guangzhou 510100, China
}

Correspondence should be addressed to Xinsheng Peng; 392439645@qq.com and Ping Zhu; tanganqier@163.com

Received 8 October 2014; Accepted 22 December 2014

Academic Editor: Jesus Simal-Gandara

Copyright (C) 2015 Yanfang Zhou et al. This is an open access article distributed under the Creative Commons Attribution License, which permits unrestricted use, distribution, and reproduction in any medium, provided the original work is properly cited.

\begin{abstract}
We applied HPLC technique to quantitatively analyze sinomenine in cubosome nanoparticles. The chromatographic method was performed by using an isocratic system. The mobile phase was composed of methanol-PBS(pH6.8)-triethylamine $(50: 50: 0.1 \%)$ with a flow rate of $1 \mathrm{~mL} / \mathrm{min}$; the detection wavelength was at $265 \mathrm{~nm}$. Sinomenine can be successfully separated with good linearity (the regression equation is $A=10835 C+1058 ; R^{2}=1.0$ ) and perfect recovery $(102.2 \%)$. The chromatograph technique was proper for quality control of sinomenine in cubosome nanoparticles.
\end{abstract}

\section{Introduction}

Sinomenine (7,8-didehydro-4-hydroxyl-3,7-dimethoxy-17methylmorphinan-6-one, chemical structure is shown in Figure 1) is an alkaloid isolated from the stem and root of Chinese medical plant Sinomenium acutum Rehd. et Wils. Because of its analgesic and anti-inflammatory effects, sinomenine has been utilized clinically to treat rheumatoid arthritis and neuralgia $[1,2]$.

In order to prevent the negative gastrointestinal adverse reactions and low bioavailability of sinomenine oral preparations, researchers have been trying to use transdermal drug delivery system to overcome these shortcomings. For example, microemulsion based gel [3], liposome [4], and ethosomes [5] had been documented in the literature. We concerned the development of sinomenine preparations for a long time and found cubosome, a novel kind of transdermal drug delivery system, could be very well suitable to the sinomenine. Cubosome and liposome both had the bilipids membrane, but cubosome showed better storing stability compared to liposomes [6]. Cubosome could also enhance the drug deposition in the skin and showed excellent skintargeted characteristics [7]. Our previous experiment has proved cubosome could enhance sinomenine skin permeation (7-fold compared to gel) and a patent was applied in China (ZL 201310087778.6). Here we describe the determination of sinomenine in cubosome nanoparticles by HPLC technique.

\section{Experiments}

2.1. Reagents and Chemicals. Glycerol monooleate (DIMODAN MO/D KOSHER, material number 116703) was kindly provided by Danisco Cultor (Brabrand, Denmark). Poloxamer 407 (PEO98POP67PEO98) was a gift from BASF (Ludwigshafen, Germany). Sinomenine was purchased from Shenzhen Yihao Technology Development Co., Ltd. (Guangdong, China). Methanol (Chromatographic Grade) was purchased from Tianjin Fuyu Chemical Co., Ltd. (Tianjin, China). Water purified through a flow water purification system (Qingdao, China) was used throughout this study. PBS ( $\mathrm{pH}$ 6.8) was made according to the Chinese Pharmacopoeia (2010). All other reagents were of analytical grade.

2.2. Apparatus. Analyses were performed on UV-6000s Spectrophotometer (Shanghai Metash Instruments Co., Ltd.) 
<smiles>COC1=CC2CN(C)C(I)(CC1=O)c1c2ccc(OC)c1O</smiles>

FIgURE 1: The structure of sinomenine.

and LC-20AT High Performance Liquid Chromatograph with SPD-20A UV-detector (Shimadzu (Suzhou) Instruments Co., Ltd).

2.3. Preparation of Sinomenine Cubosome [7, 8]. Firstly, glycerol monooleate $(2.7 \mathrm{~g})$ and poloxamer $407(0.3 \mathrm{~g})$ were first melted at $60^{\circ} \mathrm{C}$ in a hot water bath until they were homogeneous, after which sinomenine $(0.3 \mathrm{~g})$ was added to blend under continuous stirring. Water $(6.7 \mathrm{~mL})$ was then added gradually and the mixture was vortex-mixed to achieve a homogeneous state. After equilibration for at least 48 hours at room temperature, the cubic phase gel was formed. By adding $20 \mathrm{~mL}$ of water, the cubic gel was disrupted by mechanical stirring. Subsequently, the crude dispersion was fragmented for $10 \mathrm{~min}$ by intermittent probe sonication. The final dispersion of cubic nanoparticles was stored at room temperature for later studies.

2.4. Chromatographic Conditions. The analytical column was an Hichrom C18 column $(4.6 \times 250 \mathrm{~mm}, 5 \mu \mathrm{m}$, inner diameter, $5 \mu \mathrm{m}$ ). The mobile phase was methanol-PBS(pH6.8)triethylamine $(50: 50: 0.1 \%)$ with a flow rate of $1 \mathrm{~mL} / \mathrm{min}$, and the detection wavelength was at $265 \mathrm{~nm}$. The sample injection volume was $10 \mu \mathrm{L}$. The column temperature was maintained at $25^{\circ} \mathrm{C}$.

2.5. Preparation of Standard Solutions. Sinomenine stock solution was prepared in 50\% methanol solution and sonicated for $5 \mathrm{~min}$ to obtain stock solution concentrations of $320 \mu \mathrm{g} / \mathrm{mL}$. This solution was further diluted with $50 \%$ methanol solution to yield solutions containing 160.0, 80.0, $40.0,20.0,10.0,5.0,2.5$, and $1.25 \mu \mathrm{g} / \mathrm{mL}$.

2.6. Sample Preparation Procedure. $0.2 \mathrm{~mL}$ (approximately $200 \mathrm{mg}$ ) of cubosome nanoparticles was accurately transferred into a $10 \mathrm{~mL}$ volumetric flask, dissolved, and made up to volume with methanol. Then, the sample solutions were filtered using a $0.45 \mu \mathrm{m}$ filter membrane and $10 \mu \mathrm{L}$ aliquot was injected into the HPLC system.

\section{Results and Discussion}

3.1. Preparation of Sinomenine Cubosome. The milky coarse dispersions of phytantriol-based sinomenine cubosomes

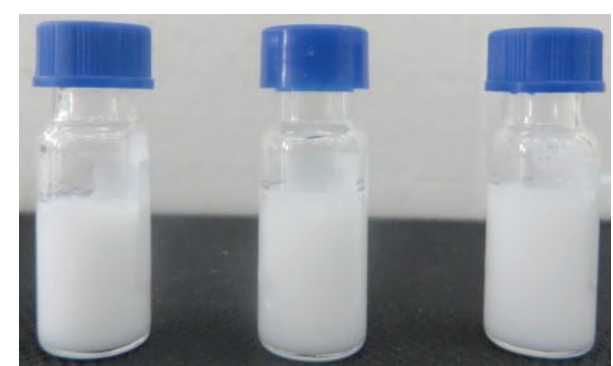

Figure 2: Photograph of sinomenine cubosome.

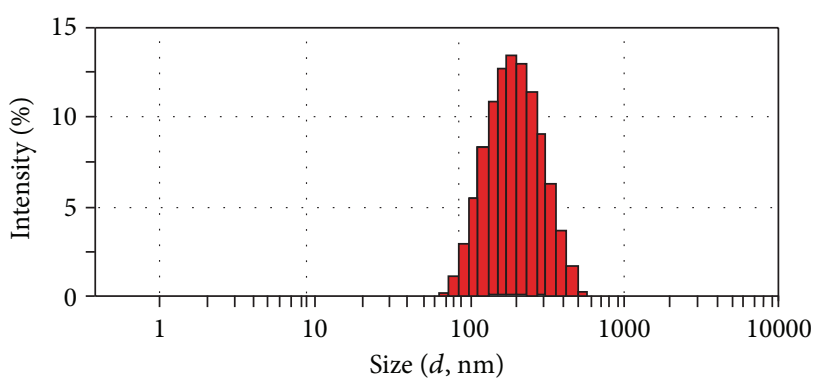

FIgURE 3: Size distribution of sinomenine cubosome.

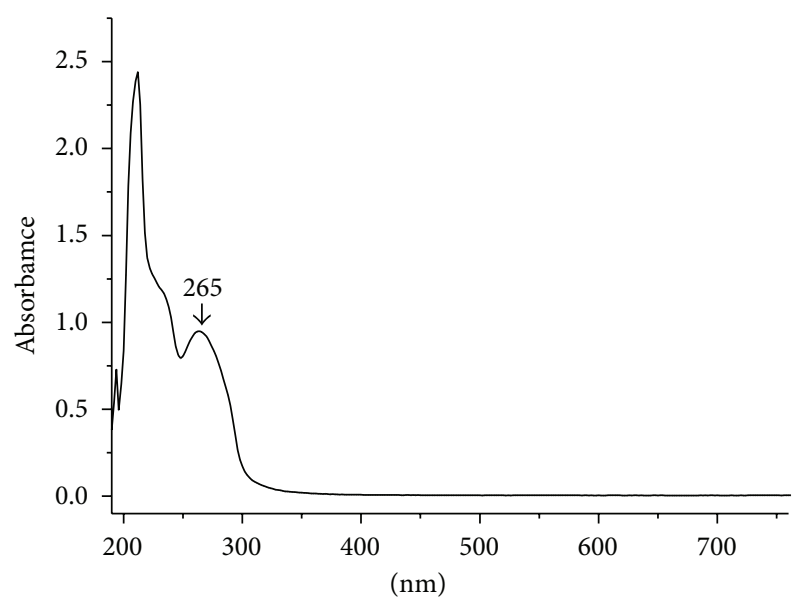

FIGURE 4: UV spectrum of sinomenine.

were obtained (Figure 2). The cubosomal particle size was determined by photon correlation spectroscopy using a ZetasizerNano ZS90 (Malvern Instruments Malvern, UK) at $25^{\circ} \mathrm{C}$. The mean $z$-average diameter and polydispersity indices (PDI) were obtained by cumulative analysis using the MALVERN software. The mean diameter of different cubosome dispersions was in an approximate range of $177.8 \mathrm{~nm}$ with the polydispersity indices (PDI) value of 0.158 (Figure 3 ).

3.2. Chromatographic Conditions. UV spectrum of sinomenine showed maximum absorbance at $265 \mathrm{~nm}$ wavelength (Figure 4). Therefore, $265 \mathrm{~nm}$ was selected as the detection wavelength. In selected spectrometry conditions, sinomenine chromatography's symmetry was with higher degrees. The 


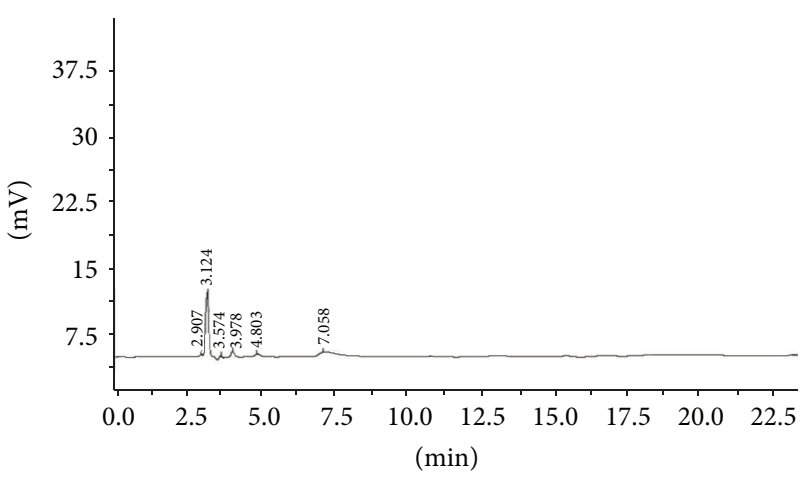

(a)

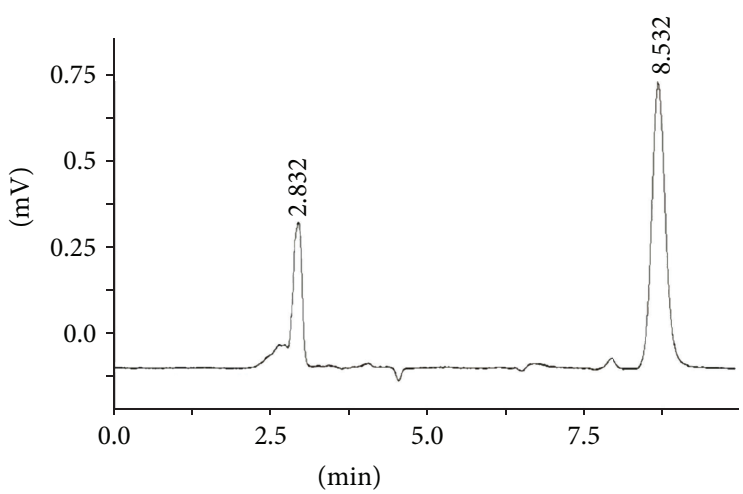

(b)

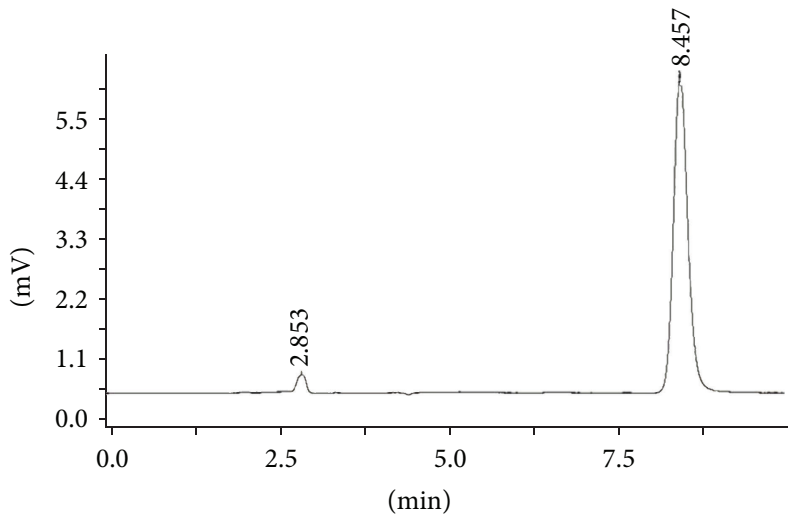

(c)

FIGURE 5: HPLC chromatogram of blank cubosome (a), standard sinomenine (b), and sinomenine cubosome (c).

TABLE 1: The intraday and the interday precision $(n=3)$.

\begin{tabular}{|c|c|c|c|c|c|}
\hline \multirow{2}{*}{ Compound } & \multirow{2}{*}{ Conc. $(\mu \mathrm{g} / \mathrm{mL})$} & \multicolumn{2}{|c|}{ Intraday } & \multicolumn{2}{|c|}{ Interday } \\
\hline & & Mean conc. $(\mu \mathrm{g} / \mathrm{mL})$ & RSD (\%) & Mean conc. $(\mu \mathrm{g} / \mathrm{mL})$ & RSD (\%) \\
\hline \multirow{3}{*}{ Sinomenine } & 100 & 101.3 & $1.33 \%$ & 101.9 & $1.51 \%$ \\
\hline & 150 & 149.6 & $1.026 \%$ & 151.8 & $1.84 \%$ \\
\hline & 200 & 200.9 & $1.014 \%$ & 199.6 & $1.36 \%$ \\
\hline
\end{tabular}

response was high and the retention time was less than $10 \mathrm{~min}$ with good reproducibility.

3.3. Specificity. Blank cubosome sample was prepared according to Section 2.3 (no sinomenine), and the chromatogram was shown in Figure 5(a). A certain concentration of sinomenine standard solution and sinomenine cubosome sample solution were injected into the HPLC system with the same operation, and the chromatogram was shown in Figures 5(b) and 5(c). The retention time was $8.53 \mathrm{~min}$. Obviously, the peaks of the sinomenine were well separated and were not affected by the excipients.

3.4. Linearity. The calibration curves for sinomenine were found to be linear within the range of 1.25 to $320.0 \mu \mathrm{g} / \mathrm{mL}$. The regression equation was $A=10835 C+1058\left(R^{2}=1.0\right)$, where $A$ is peak area and $C$ is the concentration $(\mu \mathrm{g} / \mathrm{mL})$ of sinomenine standard solution. The correlation coefficient indicated a good linear relationship between peak area and concentration over a wide range.

3.5. Precision. Precision was demonstrated at 3 concentration levels in intraday and interday studies. Intraday precision was determined by injection of sinomenine standard solutions on the same day. Interday precision was checked by repeating the studies on two different days. The intraday and the interday precisions of sinomenine are summarized in Table 1. The RSD is found to be acceptable $(\mathrm{RSD}<2 \%)$.

3.6. Recovery. Mean recovery for sinomenine at different concentration levels was found to be $100.3 \pm 2.2 \%$ (RSD = $2.19 \%, n=3), 103.1 \pm 2.2 \%(\mathrm{RSD}=2.2 \%, n=3)$, and 


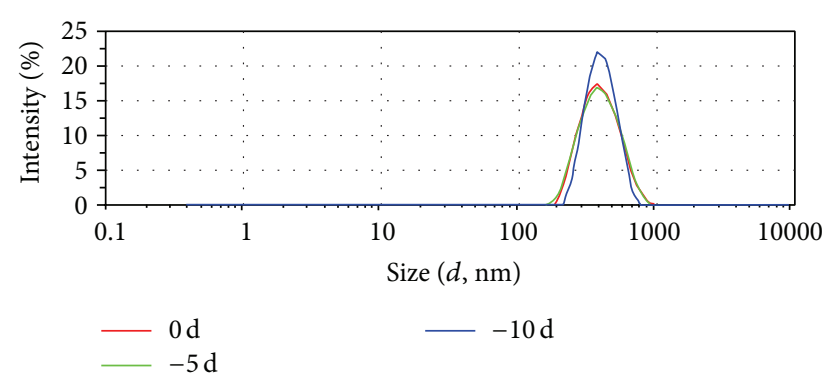

Figure 6: The influence on cubosome size distribution by high temperature test.

$103.3 \pm 1.7 \%(\mathrm{RSD}=1.6 \%, n=3)$, respectively. The low values of $\mathrm{RSD}$ revealed the present method was accurate, reliable, and reproducible.

3.7. Limits of Detection (LOD) and Quantification (LOQ). The LOD (signal/noise ratio of $3: 1$ ) and LOQ (signal/noise ratio of $10: 1)$ were determined as $7.8 \times 10^{-2} \mu \mathrm{g} / \mathrm{mL}$ and $2.6 \times$ $10^{-1} \mu \mathrm{g} / \mathrm{mL}$, respectively.

3.8. Content of Sinomenine in Cubosome Nanoparticles. Sinomenine was determined with the proposed method in cubosome nanoparticles. The mean concentration was $8.0 \mathrm{mg} / \mathrm{mL}$ $(\mathrm{RSD}=1.9 \%, n=3)$.

3.9. The Stability Experiment. The stability experiment was aimed at testing the effects during storage. The stress testing showed the cubosome was physically stable under high temperature $\left(60^{\circ} \mathrm{C}\right)$ within 10 days (Figure 6$)$. It was similar to the literatures in which cubosomes showed better storing stability at room temperature and could endure heat treatment compared to liposome [9-11]. That also provided the base for the practicability and scientificalness of the formulations and process parameters.

\section{Conclusions}

In this work, we used HPLC method to separate and determine the sinomenine in cubosome nanoparticles. The linear range of sinomenine concentration was 1.25 to $320.0 \mu \mathrm{g} / \mathrm{mL}$. Calculated by samples, the intra- and interassay precision (RSD) were less than $2 \%$. The average recovery rate was $102.3 \%$. Sinomenine peaks were well resolved and free from tailing $(<1.5)$. This method was sensitive with good precision and accuracy. The separation time was $8.53 \mathrm{~min}$ and the excipients did not interfere with the detection of sinomenine. This method was applied to sinomenine cubosome researches.

\section{Conflict of Interests}

The authors declare that there is no conflict of interests regarding the publication of this paper.

\section{Authors' Contribution}

Yanfang Zhou and Chunlian Guo contributed equally to this work.

\section{Acknowledgments}

The authors gratefully acknowledge the financial supports for the research: National Natural Science Foundation of China (nos. 81001643 and 81370230); Natural Science Foundation of Guangdong Province, China (nos. 2011B031700058, S2012010008187, 2013B021800073, and S2013010014009); Educational Commission of Guangdong Province, China (nos. 2013113-209 and 1057113027). They further acknowledge Danisco Company and BASF for generous gift samples.

\section{References}

[1] Y. Sun, Y. Yao, and C.-Z. Ding, "A combination of Sinomenine and Methotrexate reduces joint damage of collagen induced arthritis in rats by modulating osteoclast-related cytokines," International Immunopharmacology, vol. 18, no. 1, pp. 135-141, 2014.

[2] H. Zhou, Y. F. Wong, J. Wang, X. Cai, and L. Liu, "Sinomenine ameliorates arthritis via MMPs, TIMPs, and cytokines in rats," Biochemical and Biophysical Research Communications, vol. 376, no. 2, pp. 352-357, 2008.

[3] L. Wang, S. Zhang, L. Liu et al., "Preparation and in-vitro transdermal properties of sinomenine microemulsion-based gel," Traditional Chinese Drug Research and Clinical Pharmacology, vol. 22, no. 1, pp. 21-24, 2011.

[4] Y. Wang, Y. Wang, J.-J. Ling, and L.-L. Zhou, "Activities of transdermal penetration of sinomenine liposome patch," Chinese Journal of New Drugs, vol. 17, no. 24, pp. 2108-2111, 2008.

[5] L.-F. Yuan, W. He, and S. Chen, "Investigation on preparation and in vitro properties of sinomenine hydrochloride binary ethosomes," Journal of Shanxi Medical University, vol. 43, no. 9, pp. 658-661, 2012.

[6] X. Pan, K. Han, X. Peng et al., "Nanostructed cubosomes as advanced drug delivery system," Current Pharmaceutical Design, vol. 19, no. 35, pp. 6290-6297, 2013.

[7] X.-S. Peng, Y.-F. Zhou, K. Han, L.-Z. Qin, and C.-B. Wu, "Preparation and in vitro study on diffusion of capsaicin cubosome," China Journal of Chinese Material Medica, vol. 39, no. 4, pp. 644-647, 2014.

[8] X. Peng, B. Li, M. Hu et al., "Quantitative Analysis of Matrine in liquid crystalline nanoparticles by HPLC," Journal of Analytical Methods in Chemistry, vol. 2014, Article ID 368682, 4 pages, 2014.

[9] P. T. Spicer, K. L. Hayden, M. L. Lynch, A. Ofori-Boateng, and J. L. Burns, "Novel process for producing cubic liquid crystalline nanoparticles (cubosomes)," Langmuir, vol. 17, no. 19, pp. 57485756, 2001.

[10] J. Barauskas, M. Johnsson, F. Joabsson, and F. Tiberg, "Cubic phase nanoparticles (Cubosome): principles for controlling size, structure, and stability," Langmuir, vol. 21, no. 6, pp. 25692577, 2005. 
[11] S. Salentinig, A. Yaghmur, S. Guillot, and O. Glatter, "Preparation of highly concentrated nanostructured dispersions of controlled size," Journal of Colloid and Interface Science, vol. 326, no. 1, pp. 211-220, 2008. 

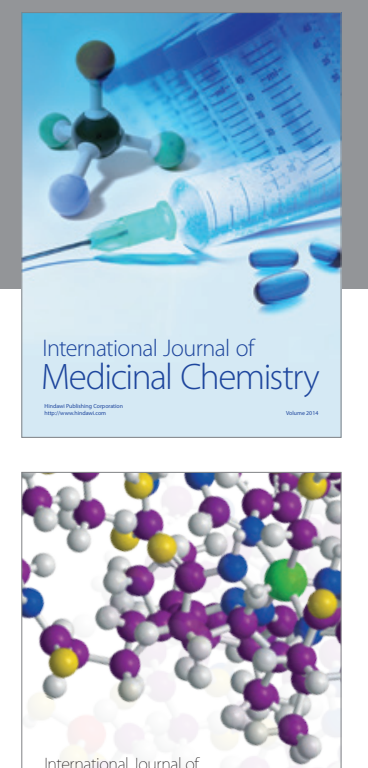

\section{Carbohydrate} Chemistry

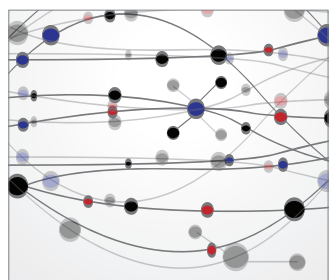

The Scientific World Journal
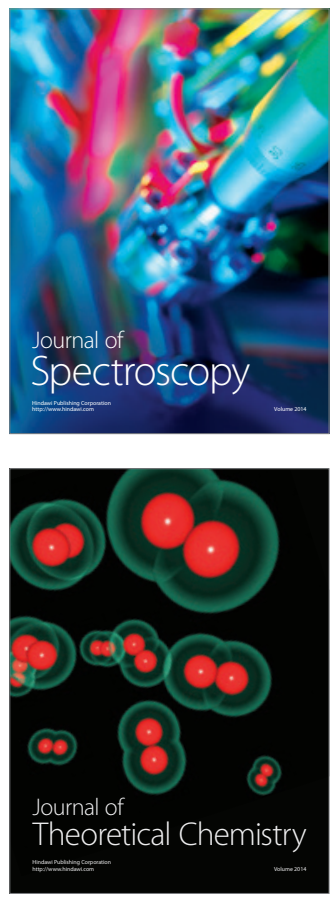
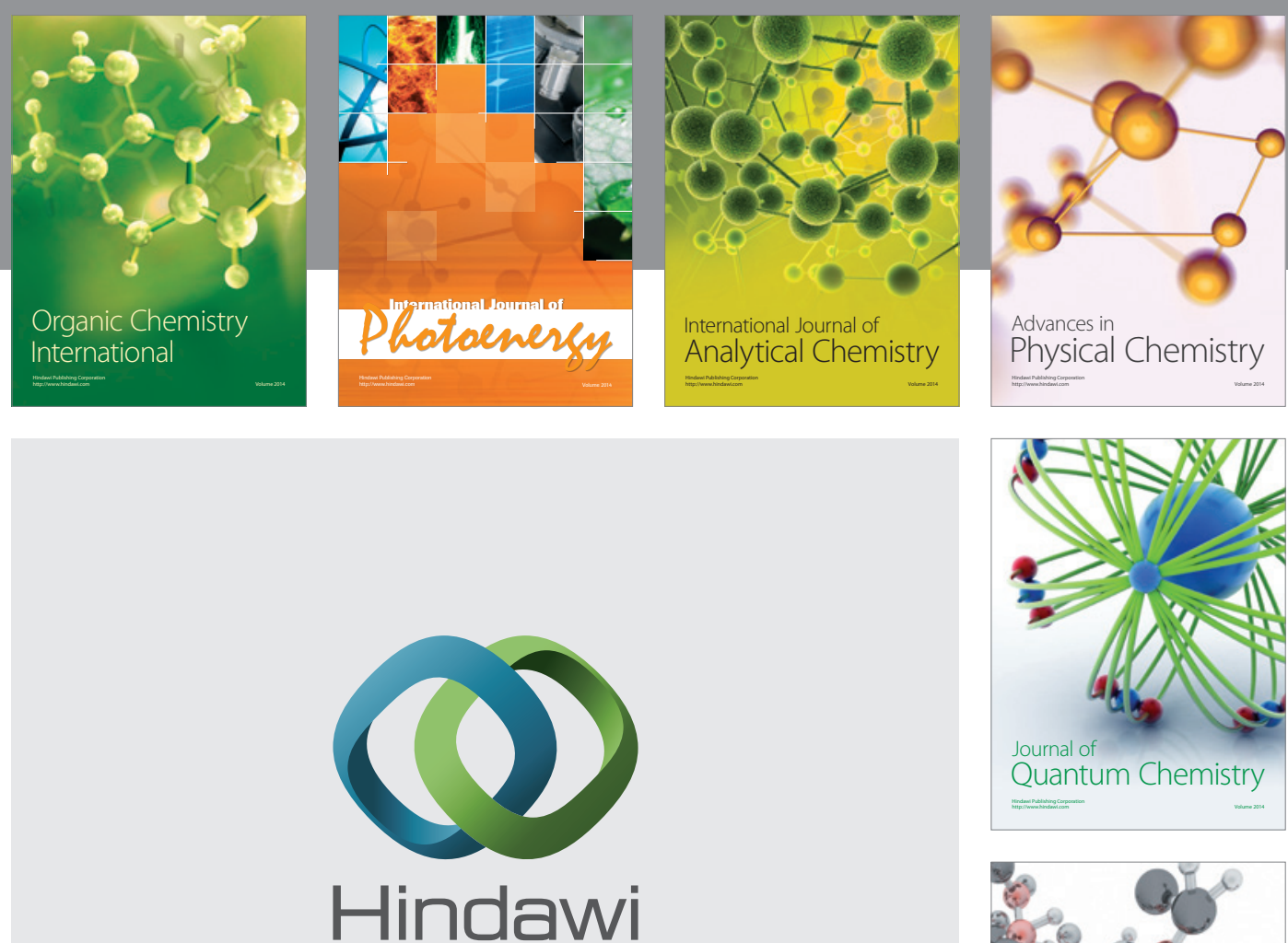

Submit your manuscripts at

http://www.hindawi.com

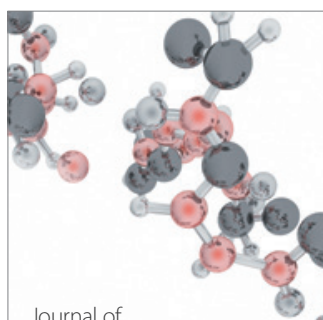

Analytical Methods

in Chemistry

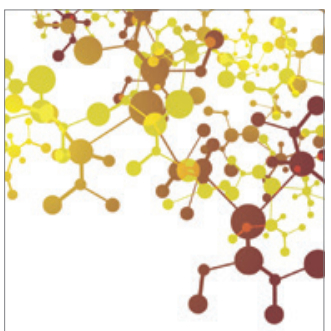

Journal of

Applied Chemistry

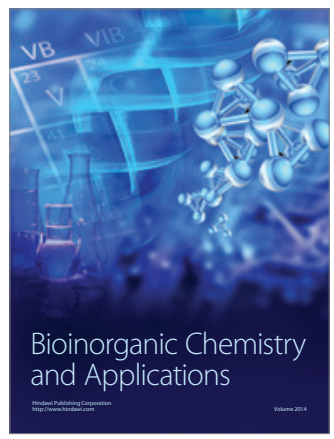

Inorganic Chemistry
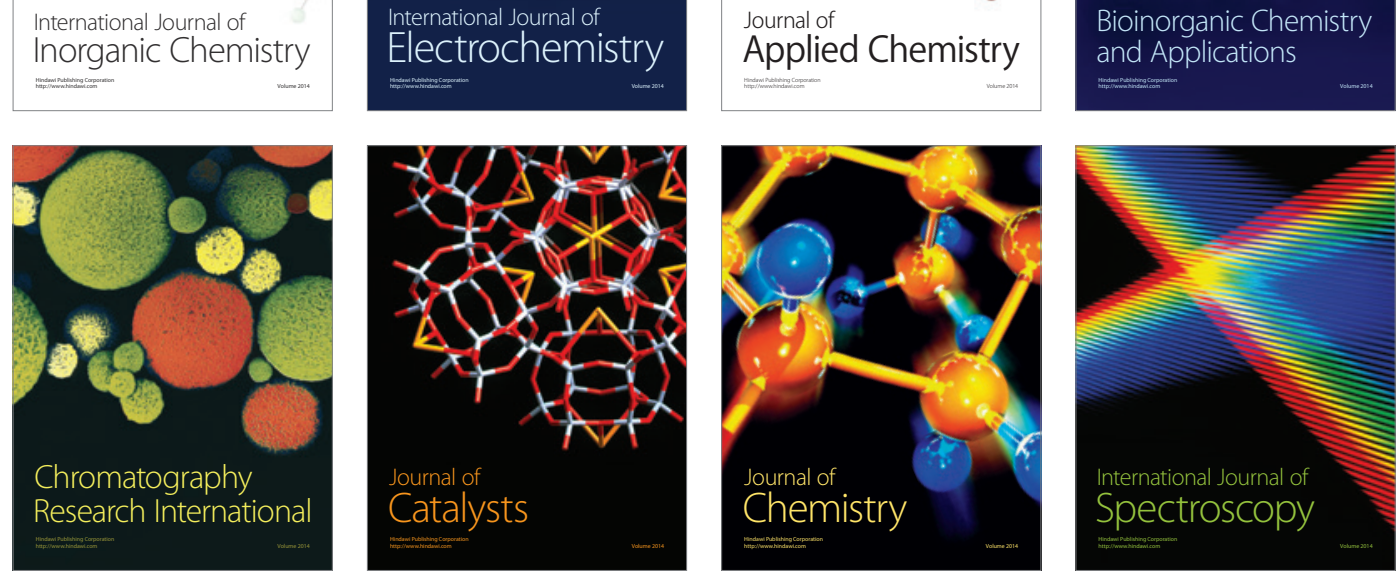